\title{
The Fc Receptor Gene Asterias Rubens: Bioinformatic Data
}

Michel Leclerc*

Immunology of Invertebrates, Orléans University (FRANCE)

"Corresponding author: Michel Leclerc, Immunology of Invertebrates, Orléans University (FRANCE)

Received date: 25 January, 2022 |

Accepted date: 5 February, 2022 |

Published date: 8 February, 2022

Citation: Leclerc M. (2022) The Fc Receptor Gene Asterias Rubens: Bioinformatic Data. J Virol Viral Dis 2(1): doi https://doi.org/10.54289/JVVD2200105

Copyright: (๑) 2022 Leclerc M. This is an open-access article distributed under the terms of the Creative Commons Attribution License, which permits unrestricted use, distribution, and reproduction in any medium, provided the original author and source are credited.

\begin{abstract}
In the present report, Asterias rubens Fc DNA Sequence was analysed from its transcriptome in bioinformatics; Identities occurred with other sea stars such as Patiria miniata and specially with mammals' proteins. Identities with Fc receptor mammal IGE was found.
\end{abstract}

\section{Introduction:}

The aim of this work is to analyse Fc DNA sequence which was discovered in 2016 [1] from

Starting material (dna) sequence in 5'-3':

TCCATTAGGGCAATGAGTGGGACTGCGCGGCTTGG

CACAGATCATCCCTTTTCTATCACGACACCTCGAGT

CTTTCCACTTGCCGTTGCTAATCTGTAATGCCACAC AGTTATTCTCCAATGATTCGACTCCAGACAGCTCAG TTTGCTCTTCTTCGATGAAGTTCGTGTAGTTGACGG GGGAATCGTTTGACCATTTCCAATCGCTTTCGTTGT GTGTATCATGGAGCCCGATCCACACGTCCCTGTCA ATTAGGTCGGTAAGAAAATCATTAATTTCTTGGTCA GTGATGGCGACCAGCCTAGCGCCGTCGTATTTAGT GCACTTCTGTTCAGCATCGACCCAGCGTGCTACATC GTCTGGAATCCAGAAGCATTCATCACGGAAGAGAT GGCCGTTGTTTAGGCAGTACTGTGGTTGACCAC
GTACTGTTTGAAGAAGATGAGCTGACCCAATAACC ATCATCATCACGAATGGAATCATTGTGAATTTGTTT GAGATACGTCCGATACGTCCGTCCGTAGATGAAAA AACTGCCGAAGTCTCTCACATAATTCCACCAGGCA TTGTTGATGCCTTGCTGCTCTATGGTTGATGCTTGG TGGCAGTCCACGAAAGAATGTGCAGTTAGGGAAAG TCCAGCTTGTATATCTC

Bioinformatics data were performed according MarchlerBauer et al [2-4].

\section{Results:}

1. Blastn original sequence: Data base: Standard data bases (nr mainly.)

Optimization: we used highly similar sequences (mega blast) We recall that molecule type was dna; the query length of 654 2 sequences were selected as shown in the table1: significant aligments were found. 
Table 1: Significant aligments from A. rubens

\begin{tabular}{|l|l|l|l|l|l|l|l|l|}
\hline Description Scientific name & $\begin{array}{l}\text { Max } \\
\text { score }\end{array}$ & $\begin{array}{l}\text { Total } \\
\text { score }\end{array}$ & $\begin{array}{l}\text { Query } \\
\text { cover }\end{array}$ & $\begin{array}{l}\text { E. } \\
\text { Value }\end{array}$ & $\begin{array}{l}\text { Per. } \\
\text { Ident }\end{array}$ & $\begin{array}{l}\text { Acc Len } \\
\text { Accession }\end{array}$ \\
\hline $\begin{array}{l}\text { Predicted: Asterias rubens macrophage } \\
\text { mannose } \\
\text { (LOC117293835) mRNA }\end{array}$ Asterias rubens & 1197 & 1197 & $100 \%$ & 0.0 & $99.69 \%$ & 1735 \\
\hline $\begin{array}{l}\text { Asterias rubens genome assembly, } \\
\text { chromosome 8 }\end{array}$ & Asterias rubens & 418 & 1162 & $96 \%$ & $4 \mathrm{e}-112$ & $10.00 \%$ & 21693562 & $\begin{array}{l}\text { LR699099. } \\
1\end{array}$ \\
\hline
\end{tabular}

The corresponding graphic summary is the following:

\section{Distribution of the top 5 Blast Hits on 2 subject sequences}

\begin{tabular}{|c|c|c|c|c|c|c|}
\hline I & ${ }_{100}^{100}$ & ${ }_{200}^{1}$ & $\mathbf{3 0 0}_{300}^{\text {Qu }}$ & ${ }_{400}^{1}$ & 500 & ${ }_{600}^{1}$ \\
\hline
\end{tabular}

\section{BlastX original sequence:}

The query length is 654in this DNA molecule: non-redundant protein sequences were used as Database (nr).
Sequences producing significant aligments: more than 100 sequences were resumed in Table 2.

Table 2: Aligment comparisons between Asterias rubens and Patiria miniata (Asterids-Echinodermata)

\begin{tabular}{|l|l|l|l|l|l|l|l|l|}
\hline Description & $\begin{array}{l}\text { Scientific } \\
\text { name }\end{array}$ & $\begin{array}{l}\text { Max } \\
\text { score }\end{array}$ & $\begin{array}{l}\text { Total } \\
\text { score }\end{array}$ & $\begin{array}{l}\text { Query } \\
\text { cover }\end{array}$ & $\begin{array}{l}\text { E. } \\
\text { Value }\end{array}$ & $\begin{array}{l}\text { Per. } \\
\text { Ident }\end{array}$ & $\begin{array}{l}\text { Acc } \\
\text { Len }\end{array}$ & $\begin{array}{l}\text { Accession } \\
\text { Macrophage mannose receptor 1-like } \\
\text { [Asteria rubens] }\end{array}$ \\
$\begin{array}{l}\text { Asterias } \\
\text { rubens }\end{array}$ & 342 & 342 & $73 \%$ & $1 \mathrm{e}-112$ & $99.38 \%$ & 510 & XP_033632182.1 \\
isoform X3 [Patiria miniata] & $\begin{array}{l}\text { Patiria } \\
\text { miniata }\end{array}$ & 130 & 203 & $75 \%$ & $3 \mathrm{e}-31$ & $39.18 \%$ & 529 & XP_038063329.1 \\
\hline $\begin{array}{l}\text { Macrophage mannose receptor 1-like } \\
\text { isoform X2 [Patiria miniata] }\end{array}$ & $\begin{array}{l}\text { Patiria } \\
\text { miniata }\end{array}$ & 129 & 201 & $65 \%$ & $1 \mathrm{e}-30$ & $46.32 \%$ & 537 & XP_038063328.1 \\
\hline $\begin{array}{l}\text { Macrophage mannose receptor 1-like } \\
\text { isoform X1 [Patiria miniata] }\end{array}$ & $\begin{array}{l}\text { Patiria } \\
\text { miniata }\end{array}$ & 129 & 201 & $65 \%$ & $2 \mathrm{e}-30$ & $43.48 \%$ & 547 & XP_038063326.1 \\
\hline
\end{tabular}

A graphic summary is following as seen below:

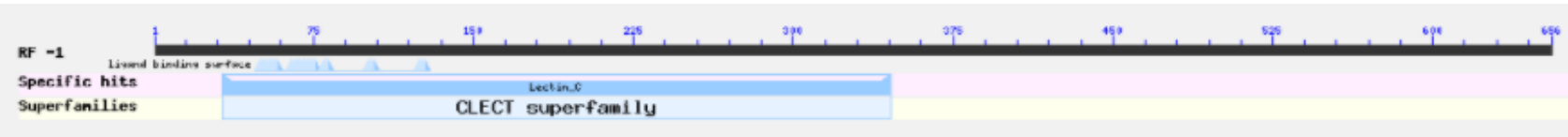

Distribution of the top 199 Blast Hits on 100 subject sequences

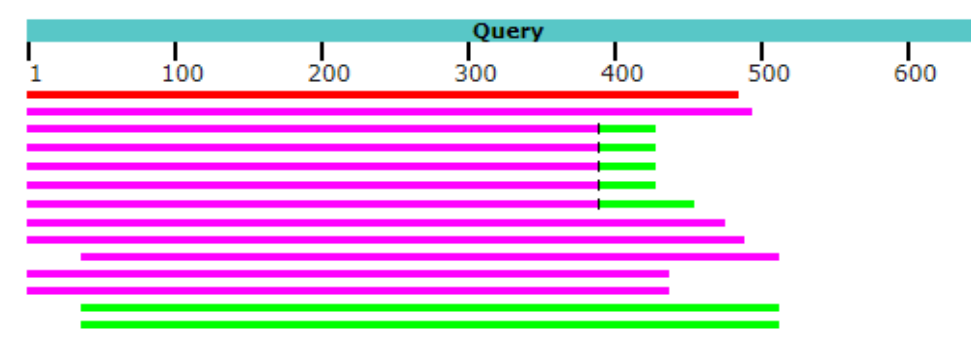


3) Putative conserved domains have been detected as shown below:

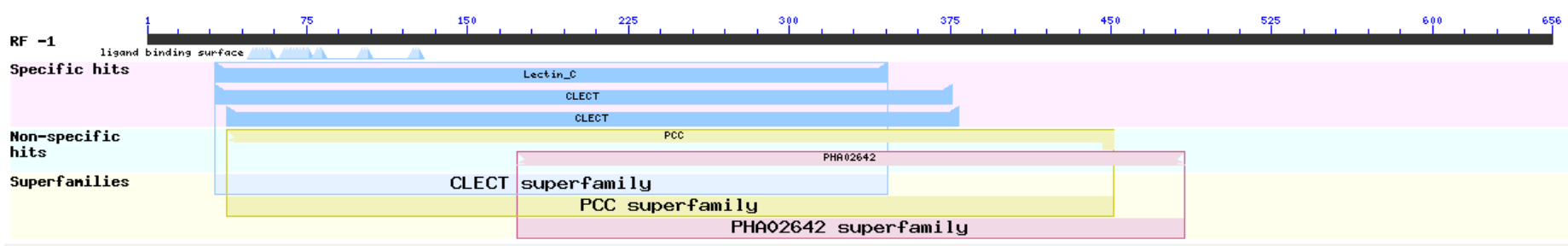

Table 3: Fc gene identities between sea star and mammals

\begin{tabular}{|c|c|c|c|c|}
\hline Name & Accession & Description & Interval & E-value \\
\hline Lectin-C & Pfam00059 & Lectin C-type domain: This family includes both ling and short form of C-type. & $31-354$ & $2.33 \mathrm{e}-19$ \\
\hline CLECT & $\mathrm{Cd} 00037$ & 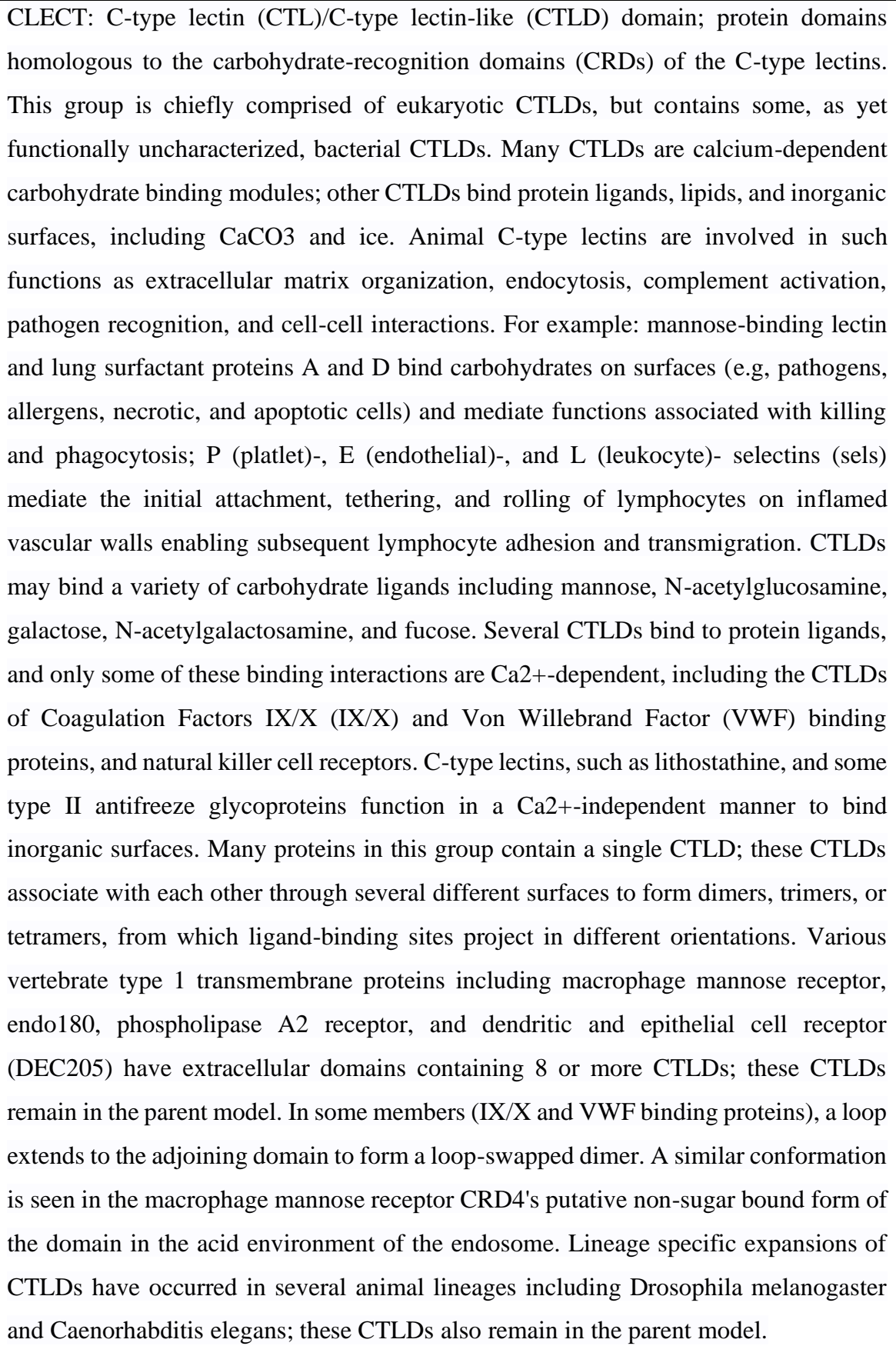 & $31-375$ & $946 \mathrm{e}-18$ \\
\hline CLECT & Smart00034 & $\begin{array}{l}\text { C-type lectin (CTL) or carbohydrate-recognition domain (CRD); Many of these } \\
\text { domain's function as calcium-dependent carbohydrate binding modules. }\end{array}$ & $37-378$ & $6.87 \mathrm{e}-15$ \\
\hline PCC & TIGR00864 & polycystin cation channel protein; The Polycystin Cation Channel (PCC) Family (TC & $37-450$ & $5.61 \mathrm{e}-08$ \\
\hline
\end{tabular}




\begin{tabular}{|l|l|l|l|}
\hline & & $\begin{array}{l}\text { 1.A.5) Polycystin is a huge protein of 4303aas. Its repeated leucine rich (LRR) segment } \\
\text { is found in many proteins. It contains } 16 \text { polycystic kidney disease (PKD) domains, one } \\
\text { LDL-receptor class A domain, one C-type lectin family domain, and 16-18 putative } \\
\text { TMSs in positions between residues } 2200 \text { and 4100. Polycystin-L has been shown to } \\
\text { be a cation (Na+, K+ and Ca2+) channel that is activated by Ca2+. Two members of } \\
\text { the PCC family (polycystin 1 and 2) are mutated in autosomal dominant polycystic } \\
\text { kidney disease, and polycystin-L is deleted in mice with renal and retinal defects. Note: } \\
\text { this model is restricted to the amino half. }\end{array}$ \\
\hline PHA02642 & PHA02642 & type lectin-like protein; Provisional. & $172-483$ \\
\hline
\end{tabular}

The Table 3 represents the identities between Asterias rubens Fc receptor and Mammal IgE Fc receptor:

$>$ Fc fragment of IgE receptor II [Rhinolophus ferrumequinum].

Sequence ID: KAF6306204.1 Length: 290

Range 1: 169 to 284

Score:73.2 bits (178), Expect:3e-11,

Method: Compositional matrix adjust,

Identities:42/119(35\%),

Positives:69/119(57\%),

Gaps:5/119(4\%).

Query

387

FRDECFWIPDDVARWVDAEQKCTKYDGARLVAIT

DQEINDFLTDLIDR-DVWIGLHDTHN 211

$\mathrm{F}++\mathrm{C}++$ + RW+ A C+K G RLV+I QE DFL I R WIGL

D+

Sbjet 169 FQRKCYYFGEGAKRWIQARLACSKLQGRLVSIHSQEEQDFLAKSIHRRGSWIGLRDLNI 227

Query

ESDWKWSNDSPVNYTNFIEEEQTELSGVESLENNC

VALQISNGKWKDSRCRDR-KGMIC 37

$\mathrm{E} \mathrm{D}+\mathrm{W}+++\mathrm{P}++\mathrm{Y}+\mathrm{N}+\mathrm{E}+\mathrm{G} \mathbf{L}+\mathrm{CV}++\mathrm{S}+\mathrm{G}+\mathrm{W} \mathrm{D}+$

$\mathbf{C}++\mathbf{G}+\mathbf{C}$

Sbjct 228 EGDFVWMDENPLDYSNWRPGEPNDGGERGLGEDCVMM-

LSSGQWNDAFCGNQLDGWVC 284.

\section{Conclusion:}

We retain mainly identities between sea star Asterias rubens $\mathrm{Fc}$ receptor and mammal $\mathrm{Fc}$ receptor occurs as shown in table-3. Many similitudes have also been observed between A.rubens Fc gene and lectins as CTL. Analogies with macrophage mannose receptor1-like from sea stars were also found. It is interesting to note that mannose sugar binds lectins. We are not surprised to find both.

The most interesting remains, from our side, the identities with mammal Fc receptor genes in conclusion.

\section{References:}

1. Leclerc M, et al. (2016) Evidence of low affinity immunoglobulin epsilon $\mathrm{Fc}$ receptor gene in an invertebrate: The sea star Asterias rubens. Clin. Res. Trials. 2(2): 152-153.

2. Marchler-Bauer A, et al. (2017) CDD/SPARCLE: functional classification of proteins via subfamily domain architectures. Nucleic Acids Res. 45(D1): 200203.

3. Marchler-Bauer A, et al. (2015) CDD: NCBI's conserved domain database. Nucleic Acids Res. 43(D): 222-226.

4. Marchler-Bauer A, et al. (2011) CDD: a Conserved Domain Database for the functional annotation of proteins. Nucleic Acids Res. 39(D): 225-229. 